\title{
Agent-Based Modeling and Simulation for the Design of the Future European Air Traffic Management System: The Experience of CASSIOPEIA
}

\author{
Martin Molina, Sergio Carrasco, and Jorge Martin \\ Department of Artificial Intelligence \\ Technical University of Madrid \\ Campus de Montegancedo S/N 28660 Madrid, Spain \\ martin.molina@upm.es, \{scarrasco,jmartin\}@fi.upm.es
}

\begin{abstract}
The SESAR (Single European Sky ATM Research) program is an ambitious research and development initiative to design the future European air traffic management (ATM) system. The study of the behavior of ATM systems using agent-based modeling and simulation tools can help the development of new methods to improve their performance. This paper presents an overview of existing agent-based approaches in air transportation (paying special attention to the challenges that exist for the design of future ATM systems) and, subsequently, describes a new agent-based approach that we proposed in the CASSIOPEIA project, which was developed according to the goals of the SESAR program. In our approach, we use agent models for different ATM stakeholders, and, in contrast to previous work, our solution models new collaborative decision processes for flow traffic management, it uses an intermediate level of abstraction (useful for simulations at larger scales), and was designed to be a practical tool (open and reusable) for the development of different ATM studies. It was successfully applied in three studies related to the design of future ATM systems in Europe.
\end{abstract}

\section{Introduction}

Air traffic management (ATM) is an example of a complex system with a large number of interacting, heterogeneous individuals (e.g., airlines, air traffic controllers, regulatory entities, passengers, etc.) operating in an intricate and constrained environment (air routes, time schedules, international regulations, etc.). The study of the behavior of ATM systems is important for the development of new methods of improving air transportation performance aspects such as safety, transport capacity, cost efficiency and environmental concerns.

Agent-based modeling and simulation was successfully applied in a number of studies of ATM systems. In recent years, important initiatives have been developed to 
modernize ATM systems. For example, the SESAR (Single European Sky ATM Research) program is an ambitious research and development initiative funded by the European Union, Eurocontrol and industry. The ultimate goal of SESAR is to develop a future ATM system for Europe, ensuring the safety and fluidity of air transport over the next thirty years, making flying more environmentally friendly and reducing the costs of air traffic management [14].

The purpose of this paper is to present the results of our research work under the CASSIOPEIA project (Complex Adaptive Systems for Optimization of Performance in ATM) that we developed in the context of the SESAR program. First, we identify new agent-based modeling challenges for the design of future ATM systems and subsequently present an agent-based approach that we designed for these challenges. Using this approach, we developed a solution to simulate new collaboration strategies of ATM stakeholders in large geographic areas at an intermediate level of abstraction that is between the microscopic and macroscopic level. Our approach was developed as a practical tool that is open and reusable for different ATM problems, and it was applied successfully in three different ATM studies.

\section{Modeling Challenges for the Design of Future ATM Systems}

According to Eurocontrol (European Organization for the Safety of Air Navigation), air traffic management (ATM) concerns the processes, procedures and resources that are involved in ensuring that aircraft are safely guided from their origin to their destination [9]. An ATM system is composed of three main, complementary sub-systems: (1) airspace management (AM), which establishes the best aerial routes for an increasing number of flights; (2) air traffic flow management (ATFM), which matches the flights with the available capacity and (3) air traffic control (ATC), which manages aircraft as they fly and move in the airports.

In general, ATM is a complex, socio-technical system [3], where different stakeholders such as traffic controllers, airport authorities, slot coordinators and air navigation service providers interact with technical systems (navigation aids, runways, taxiways, etc.) that create a social context in which the system operates. Modern ATM is more complex because new organization rules and communication mechanisms create new, geographically dispersed, collaborative decision making processes and involve several stakeholders with their own set of business behaviors (driven by self-interests and partial responsibilities). Distributed decisions exist at multiple levels (strategic, tactical and operational) that follow different temporal and spatial scales. In addition, ATMs operate as adaptive systems, able to self-organize when external conditions change (e.g., airports and airlines can modify transport routes during traffic disruptions). The European ATM system currently manages approximately 26,000 flights daily in a complex network. Since 1989, air traffic flows have increased by $33 \%$, and they are expected to nearly double over the next 20 years [9].

The study of the behavior of ATM systems using modeling and simulation tools can help in the development of new methods of improving air transportation 
performance. Because of their significant complexity, it is difficult to understand and predict the behavior of ATM systems at a macroscopic scale. The agent-based approach was successfully applied as a solution for developing models that capture air traffic decisions and interactions with an adequate level of detail. After initial studies were performed on cooperation strategies for collision avoidance [4] and real-time traffic control [13], agent-based models gained popularity as an effective approach to modeling and simulating specific dynamics of ATM systems.

Different agent-based approaches were applied in ATM studies concerning air traffic control (ATC). These agent-based models represent air traffic processes such as vehicle trajectories, collision avoidance, airport operation, etc. In this category of models, we can mention Air MIDAS [11][12], ACES [10][18], AgentFly [15] and tools such as AirTop [2] and CAST [6]. These approaches are capable of simulating how air traffic controllers manage aircraft as they fly and move in the airports with a high level of detail. For example, Air MIDAS simulates the behavior of the final approach of aircraft in the terminal airspace and their interaction between the pilots and the flight controllers for risk evaluation.

ATM studies related to air traffic flow management (ATFM) have recently been performed using also agent-based approaches. In general, they simulate ATFM on the day of the flight [1][5][17]. For example, IMPACT [5] simulates the airlines' decisions when meteorological conditions affect flight scheduling, and those conditions result in a constraint on the capacity of some airports. However, studies for future ATM systems must also consider new ATFM processes (e.g., far in advance of the day of the flight). These studies normally require the implementation of new algorithms for collaborative human decision making related to planning and scheduling (see our approach in CASSIOPEA below).

The agent-based models of ATM studies cover different temporal and geographical extensions. In general, the existing models cover a small number of hours or one day of flight in a limited number of airports (five or ten airports, a few dozen at the most). The models are defined at a microscopic level and consider details such as aircraft approach, runways, taxiways, aircraft movements and, occasionally, bus movements, passenger behavior during check-in, etc. Therefore, developing models for large areas with this level of detail requires significant effort and significant amounts of data that are sometimes not available. For the design of a new ATM system in Europe, it is important to understand cause-effect relations between policy decisions and their impact on a large scale. Therefore, it is necessary to use new representations with less detail to model and efficiently simulate areas with larger extensions, taking into account the partial availability of data.

The design of a future ATM system requires the active participation of the research community to conduct several different ATM studies. To promote this, it is important to provide practical tools (e.g., software tools and methodologies) that are easy to use and are open to the community. A number of existing approaches were designed and used as general, reusable tools for different studies (ACES, AirTop and CAST). They provide reusability using libraries of agent classes (ACES) or user interfaces for model configuration using parameter values (AirTop and CAST). In addition to this type 
of tool, it is also important to develop more practical (open and reusable) solutions that decrease the effort and cost requirements of the studies.

In summary, based on the general goals of the SESAR program in Europe, we identified the following new challenges in agent-based modeling and simulation for the design of future ATM systems: (1) modeling new decision levels in ATM systems (such as strategic decisions related to flow and capacity management with longer temporal horizons), (2) designing new representation methods to simulate at a larger scale (e.g., multinational geographic areas in Europe) taking into account limitations concerning existing data, and (3) creating new practical tools (more easily available to the research community) to support the development of new ATM studies. In the following sections, we describe our agent-based approach in CASSIOPEIA that we designed that addresses these challenges.

\section{The Agent-Based Approach in CASSIOPEIA}

CASSIOPEIA (Complex Adaptive Systems for Optimization of Performance in ATM) is a project of the SESAR (Single European Sky ATM Research) program of Eurocontrol. The general goal of the CASSIOPEIA project was to propose a modeling approach, using techniques of complex systems and paradigms of computer science, that could provide policy-makers with the means to understand and explore initiatives that affect complex ATM networks, allowing them to test potential concepts, regulations and mechanisms to manage delay propagation, capacity limits, network congestion, and other ATM phenomena.

This project was envisioned as a solution to facilitate an understanding of the cause-effect relation between policy decisions in different sectors of aviation and air traffic performance for different scopes and scales of application of regulations. As a result of the project, an agent-based modeling approach was designed, and it was successfully applied in several studies regarding various regulations in the context of European ATM. In the following, we describe the characteristics of our agent-based model and discuss the results obtained in different studies.

\subsection{The Agent-Based Model}

The model of CASSIOPEIA includes agents corresponding to different ATM stakeholders. For example, there are agents such as network managers, airlines (with agent subclasses: network airline, cargo airline, low-cost airline, etc.), airports (with information such as geographic location, category, etc.), and aircraft (with information such as model, capacity, CO emissions, weight, etc.). The model also includes objects related to the environment and general decision-making processes such as flight plans, time slots, and geographical sectors. The agent models follow a BDI approach, with beliefs, goals and plans. Figure 1 shows a partial example of a model for an airline agent, showing the components related to the rescheduling of the network operation plan. 


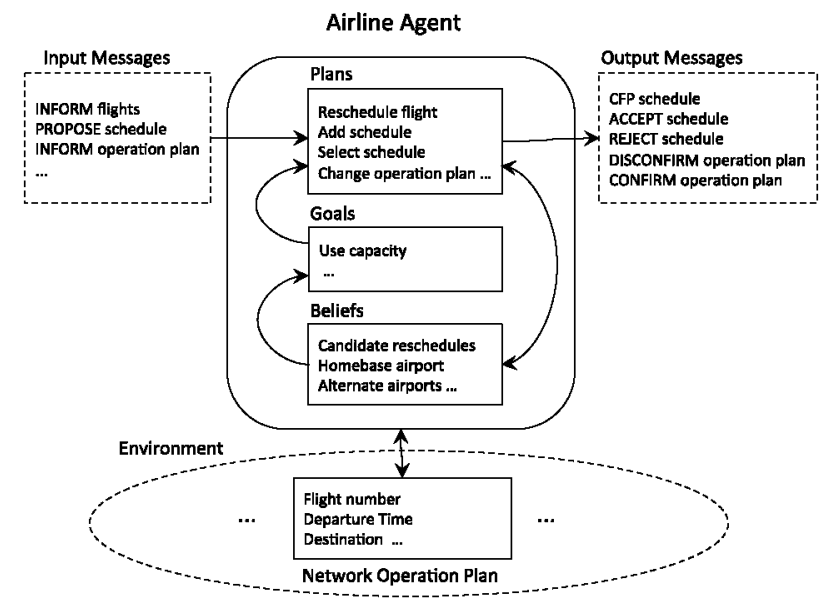

Fig. 1. Summary of the model of the airline agent (related to flight reschedule)

In this model, we implemented algorithms for collaborative decision-making processes corresponding to future ATM systems. For example, we simulate how airports interact with airlines and other airports to propose new schedules for flights (e.g., a new arrival time or new destination). The airline agent can receive input messages from other agents (for example, an airport that proposes a new flight schedule) and the airline uses local decision rules to select the best proposals. Figure 2 summarizes an example of an interaction to establish a new departure time for a flight (numbers in parentheses describe the temporal order of messages). First, the airport $E G L L$ interacts with the airport WSSS to determine a new departure time for a flight that must be rescheduled. Then, the airline $B A W$ receives the new arrival times proposed by two airports ( $E G L L$ and $E G S S)$. Finally the airline accepts one of the proposals and rejects the other one.

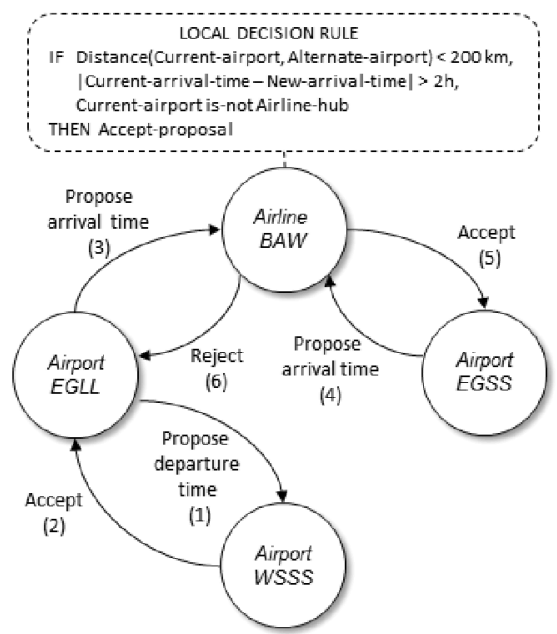

Fig. 2. Partial example of agent interaction during collaborative decision making 
In the CASSIOPEIA model, we also implemented algorithms that simulate how airlines interact to bid and sell air traffic slots to reschedule flight plans with lower costs. We implemented various bidding-selling strategies based on new studies of coordination [7]. Airlines use local sets of decision rules to accept or reject bidding proposals. An example of a rule is if $\left[\operatorname{Cost}\left(s_{p}\right)-q \cdot k\right]<\operatorname{Cost}\left(s_{r}\right)$ then $A$ ccept $(b)$, where $s_{p}$ is the provided slot by the bidder; $s_{r}$ is the requested slot by the seller; $q$ is the amount of money provided by the bidder; $\operatorname{Cost}(x)$ is the estimated $\operatorname{cost} ; k$ is a constant between 0 and 1 , whose value depends on the selected strategy; and $b$ is the bid proposal.

We implemented algorithms to simulate certain air traffic processes following an intermediate level of abstraction using a stochastic approach. Instead of modeling all the precise details, which is not possible for practical reasons, a number of components are not explicitly modeled, and only the outcomes are estimated using probability distributions. For example, we follow this approach to simulate how airlines coordinate aircrafts in the presence of delays. The model of the airline agent includes plans (using decision rules) to determine how to change the behavior of aircrafts to recover delays. For example, the airline decides that an aircraft, with a delay of 23 minutes, must reduce its delay to 10 minutes. To do this, the airline calculates a new cost index for the aircraft (a number used by the aircraft to adjust its speed during the flight) and sends a message to the aircraft with the new cost index. We simulate the movement of aircraft between airports using a stochastic approach (Figure 3).

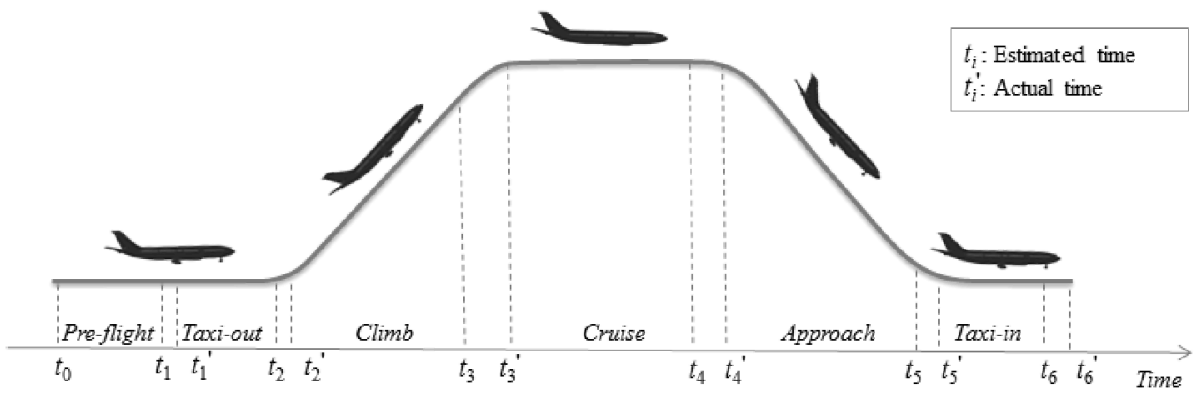

Fig. 3. Simulated events corresponding to the different phases of a flight

The model of the aircraft agent includes a plan that simulates the different phases of flights. The phases are represented as a set of events $E=$ \{pre-flight, taxi-out, climb, cruise, approach, taxi-in $\}$. According to this plan, the actual time for an event is generated as $t_{i}^{\prime}=t_{i}+\Delta t_{i}^{d}+\Delta t_{i}^{n}$, where $t_{i}$ is the estimated planned time of event $e_{i}$, $\Delta t_{i}^{d}$ is the accumulative delay for the event $e_{i}$, and $\Delta t_{i}^{n}$ is a noise value. The value for $\Delta t_{i}^{n}$ is generated automatically as a random number following the probability distribution $N\left[0,\left[\alpha\left(t_{i}-t_{i-1}\right)\right]^{2}\right]$, where $\alpha$ is a constant value (for example, $\alpha=0.015$ ). We also apply a stochastic approach to simulate other air traffic processes (e.g., the duration of certain phases of flight using a log-normal $L n N$ probability distribution).

Our software architecture in CASSIOPEIA was designed to be a general (open and reusable) tool for use in various ATM studies. The architecture integrates open-source components to facilitate reusability at reduced costs. We used the general, agentbased modeling tool Jadex for agent formalization and simulation control together 
with a relational database (MySQL) to store agent instances and simulation results. Agent models are specified using the XML declarative language, together with algorithms implemented in the Java language for agent plans, with reusable libraries of classes. The architecture includes a visualization tool, developed in this project, to visualize complex agent interactions with large numbers of messages. This tool aggregates information using different dimensions (spatial, temporal, types of messages, types of agents, etc.) and presents the information using animations on geographic maps (using OpenStreetMaps) and specific types of hierarchies and tables. For example, figure 4 shows on the left a tree of messages between airlines and airports aggregated by consecutive time points. On the right, a geographic map shows the messages corresponding to a specific simulation time.

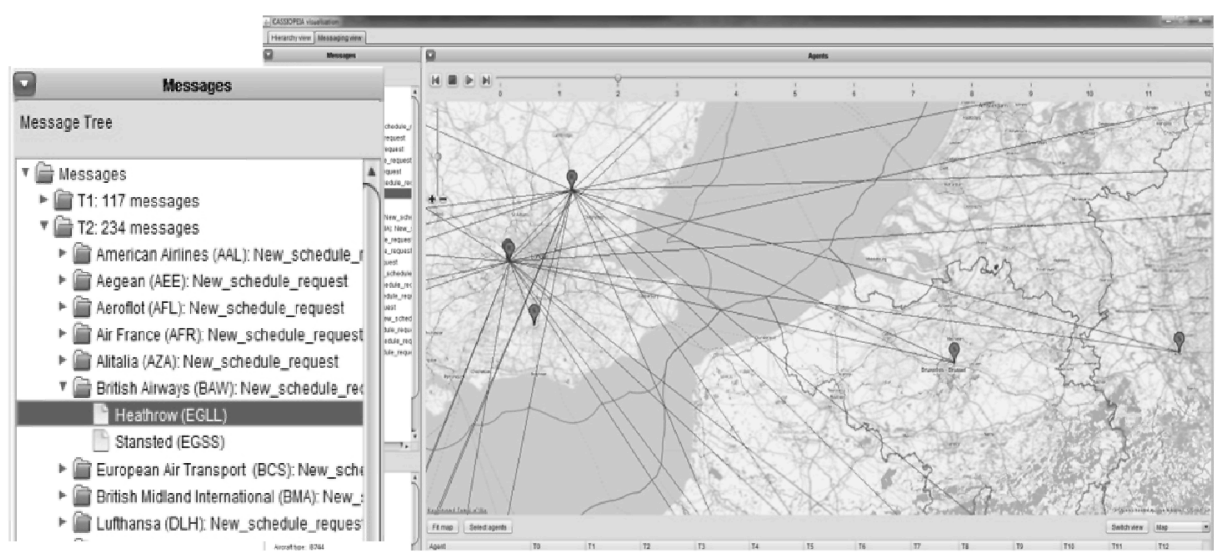

Fig. 4. Visualization tool showing agent interaction messages

\subsection{Applications}

Our agent-based approach was used in three different studies in the CASSIOPEIA project: (1) study 1: analyze the effects of new environmental regulations (e.g., restrictions of night traffic at certain airports to reduce noise pollution), (2) study 2: analyze the effect of capacity constraints considering as a new strategy that airlines can exchange traffic slots (i.e., an air traffic slot as a tradable commodity), and (3) study 3: analyze the effect of new methods of speed adjustment for aircraft based on environmental conditions.

For these studies, we used data on air transportation in Europe from several sources. The majority of the data was acquired from ALL-FT+, a dataset collected by the PRISME group from Eurocontrol that includes information on trajectories for all flights crossing the European airspace from the 1st of March to the 31st of December 2011 and includes a total of 10.3 million flights. The data was provided in a large text file (more than 350 Gbytes), and it was necessary to appropriately clean, filter, validate and store in a database. We also used data from other sources (Demand Data Repository DDR, OpenFlights, OurAirports and Geonames) to complement these datasets with additional information (geographic names, time zones, etc.). 
This data was used as input data for these studies. Each study included several simulations with different input data corresponding to various scenarios. For example, for study 1, we used data from 79,852 flights, 838 airports and 84 airlines and generated approximately 4,500 interaction messages (during one of the simulations). For study 2, we used data from 20,529 flights and 272 airlines and generated 4,938 interaction messages; and for study 3 , we used data from 114 airports and 676 flights and generated approximately 2,000 messages. The CASSIOPEIA software architecture was reused to develop the agent models for the three studies. We reused $78 \%$ of the agent specifications in the XML language, $72 \%$ of the Java code and $91 \%$ of the database design.

The data was also used to estimate certain common quantitative parameters for the different scenarios. This was done manually and with simple statistical procedures. We did not use this data to validate the simulation results (these results correspond to new traffic policies for future ATM systems). Instead, we simulated certain ATM scenarios that were similar to the scenarios used in other ATM studies, and the domain experts verified that they generated similar simulation results.
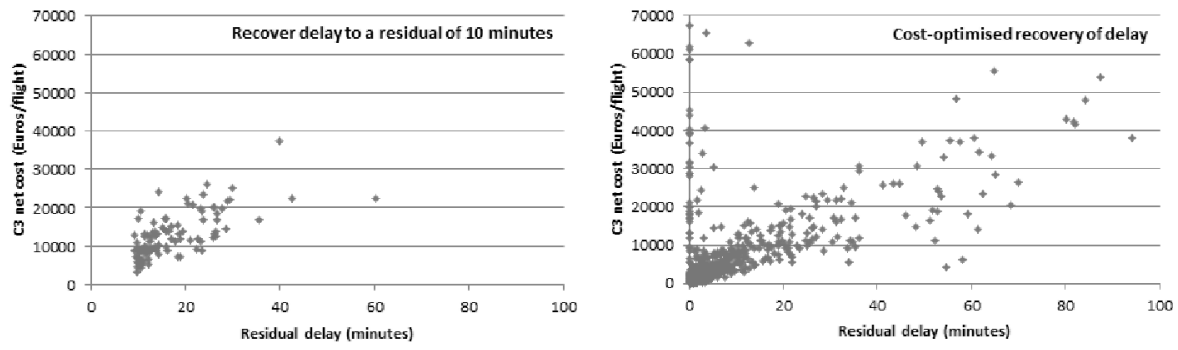

Fig. 5. Simulation results comparing two different strategies for delay recovering: (1) recover delay to a residual 10 minutes, and (2) optimum cost strategy.

The analysis of the results generated conclusions concerning the cause-effect relations in the ATM systems. Examples of the conclusions established by domain experts are as follows: traffic restrictions in the morning have worse economic effects than at night (study 1), direct slot trading between airlines can decrease costs by approximately $30 \%$ (study 2 ), and a reduction in delays is obtained when a new flight strategy for speed adjustment is applied by airlines and aircraft (the dynamic cost index strategy) (study 3).

In general, the results showed simulated effects related to complex adaptive system behavior such as system adaptation, emergent behavior and ripple effects. These cases simulated how the ATM system was able to adapt to environmental constraints using self-organization. For example, we imposed traffic restrictions at certain airports in study 1 (e.g., to reduce noise pollution at night). The simulation showed how airlines and airports could collaborate to adapt to these restrictions by finding alternative paths and new timetables and by rescheduling flight plans.

Certain behaviors of the system at a macroscopic level, which were difficult to anticipate by domain experts at this level of abstraction, emerged as a result of the 
interaction among agents and their environment. In study 1, for example, an effect emerged concerning traffic accumulation near the borders of restricted time intervals, which was stronger in the morning hours. Another example of emergent behavior is the global effect of local decision strategies concerning delay recovery. Figure 5 shows that the global delay of flights is significantly lower when the strategy of recovery delay to a residual of 10 minutes is applied instead of an optimum cost strategy. The simulations also showed ripple effects for events. In study 1 , for example, we observed how the traffic constraints at certain airports were propagated to other airports and airline schedules. Using large scale areas for the simulation (e.g., 79,852 flights corresponding to seven days and 838 airports) was important for the analysis of this type of effect.

\section{Discussion}

In the context of the SESAR European program, it is important to provide policy makers with the means to analyze initiatives that affect future ATM networks on a multinational scale in Europe. In the CASSIOPEIA project, we explored how agentbased modeling and simulation can help in this regard. In this project, we designed an agent-based approach using new methods for collaborative human decision making in air traffic flow management. We implemented methods based on new models of coordination in ATM that were based on new and more agile communication mechanisms between stakeholders (for example, bidding-selling strategies for air traffic slots).

Our representation in CASSIOPEIA follows an intermediate level of abstraction (a mesoscopic level, between microscopic and macroscopic). A number of air traffic processes were abstracted using a stochastic approach. This level of representation was useful to simulate larger geographic areas (containing hundreds of airports) and larger temporal intervals, using available partial data. The simulations using this approach generated emergent behaviors that were difficult to anticipate by domain experts on a macroscopic scale. Future research concerning models with intermediate levels of abstraction can explore the use of methods for automatic calibration. For example, uncertainty representations (e.g., bayesian networks) can capture, in aggregated models, sets of behaviors with explicit measures of uncertainty. This type of representation could be automatically calibrated using machine learning methods for certain types of agents and subsequently reused for other agents to build complex models.

In air transport, it is difficult to obtain real-world data for ATM studies for various reasons (large amounts of data for large-scale studies, private information, high cost of data acquisition, etc.). In CASSIOPEIA, we had access to the ALL-FT+ dataset on European air traffic from the PRISME group. However, we had delays in obtaining the data (e.g., receiving the appropriate permissions), and a significant amount of effort was required to clean and filter the data. In CASSIOPEIA, it was useful to obtain additional data from open sources such as OpenFlights, OurAirports and Geonames. We expect that in the future, ATM data will become more accessible with the 
help of initiatives such as Open Data (e.g., data.gov) and Linked Data with explicit standard metadata.

It is important to have practical tools (more open and reusable) and methodologies that facilitate model development for various ATM studies. In CASSIOPEIA, we implemented a software architecture following an open concept with declarative representations for ATM agents and open-source tools. We used the XML language to formulate explicit declarative definitions for agents (e.g., airlines, airports, etc.) that were reused in various ATM studies. The software architecture was developed by integrating open software tools (Jadex, MySQL, OpenStreetMaps, etc.) to facilitate reusability at reduced costs. Jadex was useful for agent model specification and simulation control, although we found certain problems (partial documentation and a number of execution errors) that should be solved in a more mature version of this tool. Future research on the reusability of agent models for ATM systems can explore the use of declarative languages for agent model specification (such as XML) in combination with standard ontologies concerning ATM systems [16]. This combination can be used to provide more usable general models with representations that are closer to the ATM domain.

In our project, we designed and implemented a visualization tool to help analyze complex agent interactions containing thousands of messages. This was useful to configure simulations and to analyze certain global behaviors (e.g., ripple effects in ATM networks). In general, it is important to have advanced visualization tools for ATM studies with large amounts of data. Future research can explore the use of data analysis methods in combination with new graphical views, which are useful for ATM studies, to generate aggregated presentations that anticipate useful displays for the user, following a mixed-initiative, user-system interaction. For example, we are currently experimenting with new graphical displays that combine new versions of AUML diagrams with agent ontologies in the ATM domain to visualize summaries of agent interactions.

\section{Conclusions}

Agent-based modeling and simulation was successfully used for ATM studies (e.g., for air traffic control problems). However, the design of future ATM systems, as it is defined by the SESAR program in Europe, presents new challenges such as modeling new strategic decision levels, developing new representation methods for large-scale simulation using partial data, and creating more practical tools to facilitate the development of studies for the research community.

In the CASSIOPEIA project, we developed an agent-based approach for these challenges and obtained several results. We formulated new algorithms for distributed-collaboration decision making for strategic decision levels and used intermediate abstraction levels for simulations using stochastic approaches. Our software architecture in CASSIOPEIA was designed as a general tool, open and reusable, for the development of different ATM studies. 
We proved the generality and applicability of our approach using three different ATM studies in the European airspace, which included strategic decisions concerning air traffic flow management and air traffic control. In comparison to previous related studies, we simulated new ATM decision processes, and they were applied to larger areas (with hundreds of airports and longer temporal scales). Our software architecture was used for the three ATM studies with high percentages of reuse (more than $70 \%$ ) of the different components.

The majority of the implemented agent models and algorithms in CASSIOPEIA are specific to the ATM domain. However, part of our design for the architecture (e.g., integration of software tools and visualization methods) could be reused to simulate complex systems with distributed decisions and geographically dispersed stakeholders in other transportation problems (road traffic, rail transport, ship management, etc.) and other domains (water management, electrical distribution management, etc.).

In the future, we plan to continue our research to provide additional solutions for the ATM challenges using new agent-based approaches (e.g., with uncertainty models with machine learning methods for large-scale, agent-based simulations) together with practical software tools and methodologies.

Acknowledgements. The research presented in this paper was carried out under the CASSIOPEIA project. This project received financial support from Eurocontrol under the SESAR program (Single European Sky ATM Research). The consortium members of the CASSIOPEIA project were the Innaxis Research Institute, the Technical University of Madrid (Department Artificial Intelligence and Department of Air Transport) and the University of Westminster (Department of Transport Studies). The authors would like to thank a member of our research group, Nataliia Stulova, for the implementation of the visualization tool.

\section{References}

1. Agogino, A.K., Tumer, K.: A multiagent approach to managing air traffic flow. Autonomous Agents and Multi-Agent Systems 24(1), 1-25 (2012)

2. Airtop: Air Traffic Optimization, http://wWw . airtopsoft. com

3. Bouarfa, S., Blom, H.A., Curran, R., Everdij, M.H.: Agent-based modeling and simulation of emergent behavior in air transportation. Complex Adaptive Systems Modeling 1(1), 126 (2013)

4. Cammarata, S., McArthur, D., Steeb, R.: Strategies of Cooperation in Distributed Problem Solving. In: Proc. 8th International Joint Conference on Artificial Intelligence, Karlsruhe, Germany (1983)

5. Campbell, K., Cooper, W., Greenbaum, D., Wojcik, L.: Modeling Distributed Human Decision-Making in Traffic Flow Management Operations. In: Third USA/Europe Air Traffic Management Research and Development Seminar, The MITRE Corporation, McLean, VA (2000)

6. CAST, Airport Research Center, http : / /Www . airport-consultants .com 
7. Castelli, L., Pesenti, R., Ranieri, A.: The design of a market mechanism to allocate air traffic flow management slots. Transportation Research Part C: Emerging Technologies 19(5), 931-943 (2011)

8. De Jonge, H., Seljée, R.: Optimisation and Prioritisation of Flows of Air Traffic through an ATM Network. NLR-TP-2011-567, NLR, Amsterdam (2011)

9. Eurocontrol: The European Organisation for the Safety of Air Navigation, http://www. eurocontrol.int

10. George, S., Satapathy, G., Manikonda, V., Wieland, F., Refai, M.S., Dupee, R.: Build 8 of the Airspace Concept Evaluation System. In: AIAA Modeling and Simulation Technologies Conference (2011)

11. Gore, B.F., Corker, K.M.: Increasing aviation safety using human performance modeling tools: an Air Man-machine Integration Design and Analysis System application. Simulation Series 34(3), 183-188 (2002)

12. Gore, B.F.: Man-machine integration design and analysis system (MIDAS) v5: Augmentations, motivations, and directions for aeronautics applications. In: Human Modelling in Assisted Transportation, pp. 43-54. Springer (2010)

13. Ljunngberg, M., Lucas, A.: The OASIS Air Traffic Management System. In: Proc. 2nd Pacific Rim Conference on AI, Seoul, South Korea (1992)

14. Sesar: Single European Sky ATM Research, http : / / www . sesarju . eu.

15. Sislak, D., Pechoucek, M., Volf, P., Pavlicek, D., Samek, J., Mark, V., Losiewicz, P.: AGENTFLY: Towards Multi-Agent Technology in Free Flight Air Traffic Control. In: Defense Industry Applications of Autonomous Agents and Multi-Agent Systems, ch. 7, pp. 73-97. Birkhauser Verlag (2008)

16. Van Putten, B., Wolfe, S., Dignum, V.: An Ontology for Traffic Flow Management. In: ATIO 2008, Anchorage, Alaska (2008)

17. Wolfe, S., Jarvis, P., Enomoto, F., Sierhuis, M., Putten, B., Sheth, K.: A Multi-Agent Simulation of Collaborative Air Traffic Flow Management. In: Bazzan, A., Klugl, F. (eds.) Multi-agent Systems for Traffic and Transportation. IGI Global Publishing (2009)

18. Zelinski, S.: Validating the Airspace Concept Evaluation System Using Real World Data. In: AIAA Modeling and Simulation Technologies Conference and Exhibit, AIAA-20056491, San Francisco, California (2005) 